\title{
The 5th International Workshop on Software Reuse and Safety
}

\author{
William B. Frakes ${ }^{1}$ and John Favaro ${ }^{2}$ \\ ${ }^{1}$ Department of Computer Science, Virginia Tech, \\ 7054 Haycock Rd., Falls Church VA 22043 \\ frakes@cs.vt.edu \\ ${ }^{2}$ Intecs SpA, Via Giannessi 5, 56100 Pisa, Italy \\ john. favaro@intecs.it
}

\begin{abstract}
There is one domain in which software reuse is looked upon with suspicion: the domain of safety critical systems. This workshop (http://www.favaro.net/john/RESAFE2011/) addresses the related issues, building upon the results of previous workshops. Updates to current activities in a number of safety-critical domains are discussed, ranging from space and railway to automotive and medical.
\end{abstract}

\section{Safety Relevant Characteristics of Reusable Software}

In her book Safeware, Leveson observes that a common problem in much current work in the area is the tendency to consider safety together with other nonfunctional properties such as reliability, availability, and dependability, leading to the impression that improvement in any of the other areas will automatically lead to improvements in its safety-related characteristics. But current standards for safety-critical software development insist on separate treatment of safety, requiring "safety lifecycles," separate safety analyses, and specific process-related roles for personnel among other issues - and special treatment of reuse in safety-critical development.

\section{Roadmap for Research in Software Reuse and Safety}

One contribution of this workshop is the identification of areas in which researchers can work to advance the state of the art with respect to reuse and safety, improving upon the roadmap developed in the preceding editions of this workshop. Current themes addressed in this edition include the relationship of software safety to architectural frameworks emerging in various safety-critical domains, as well as issues arising from the emergence of model-based development as a paradigm in many of these domains. 